\title{
EXPLORING SPATIAL THOUGHT
}

ROBERT M. KITCHIN is a lecturer in the School of Geosciences at Queen's University of Belfast, UK. He received a master's degree in geographical information systems from the University of Leicester and a Ph.D. in geography from the University of WalesSwansea. His research interests focus on human spatial behavior, cognitive mapping, the integration of theory and practice from geography and psychology, and geography and disability.

ABSTRACT: This article reports on an exploratory investigation of the different ways in which people think about geographical space and formulate answers to cognitive mapping tasks. To try to discover the strategies of spatial thought used in completing a task, designed to measure configurational knowledge (knowledge of the associations between and relative locations of places), individuals were interviewed while they undertook two of four different tests. This was followed by a debriefing interview where respondents could express their feelings and judgments concerning each test. Such an introspective methodology, although being difficult from which to draw any definitive conclusions, does allow an insight into the strategies used in spatial thought and can provide useful information concerning how traditional cognitive mapping research should develop. Many strategies of spatial thought were found to exist. These can be divided into common strategies that concern geographical knowledge (e.g., imagining a map), common task strategies that are general problem-solving strategies usable over several tests (e.g., elimination to narrow choices), and task-specific strategies constrained by the nature of a particular task (e.g., working out distance and direction between $A$ and $B, B$ and $C$ to determine $A$ to $C$ ). In addition, by comparing quantitative results with the qualitative interviews it was possible to examine whether the adoption of certain strategies led to more accurate spatial products (extemalized representation of knowledge).

Since Lynch's (1960) seminal work, researchers, predominantly from geography, planning, and psychology, have been attempting to comprehend cognitive map knowledge and abilities (see edited collections by Downs \& Stea, 1973a; Gärling \&

AUTHOR'S NOTE: I would like to thank Mark Blades and Dan Montello for comments on an earlier draft of this article, the two anonymous reviewers for helpful and sound advice and the 1994-1995 geography intake at the University of Wales-Swansea for volunteering to take part in the experiment for no reimbursement. Address correspondence and reprint requests to Robert $M$. Kitchin, School of Geosciences, Queen's University of Belfast, UK BT7 1NN, telephone $+441232273368, f a x+441232321280$, E-mail rkitchin @qub.ac.uk.

ENVIRONMENT AND BEHAVIOR, Vol. 29 No. 1, January 1997 123-156

C 1997 Sage Publications, Inc. 
Evans, 1991; Gärling \& Golledge, 1993; Mark \& Frank, 1991; Moore \& Golledge, 1976; Portugali, in press). Research centers on how individuals acquire, store, recall, and decode spatial and environmental information (Downs \& Stea, 1973b). Kitchin (1994a) reported that cognitive mapping research has theoretical utility in understanding spatial decision making, in particular with reference to wayfinding ability, migration choices, consumer behavior, and recreation decisions. In addition, cognitive maps can be of use as spatial mnemonics, for interpreting texts that describe places (Tuan, 1975), and provide information about people's attitudes toward different places (Saarinen, 1973). Cognitive mapping research has applied utility in planning and education. Information concerning how people think about and behave in different environments can be used to design environments that facilitate easier use and are more enjoyable to live in and travel through (Lynch, 1976). This has particular relevance to groups who take little active participation in the planning process, such as the disabled, the elderly, and children (Spencer, Blades, \& Morsley, 1989). In addition, if we can understand how individuals think about geographical concepts, including the everyday geographic environment and geographic materials such as maps, it may be possible to devise more effective teaching strategies to convey this information (Catling, 1978; Spencer \& Blades, 1993). Alternatively, it may be possible to design geographic information providers, such as maps and aerial images, that are easier to understand (Edwards, 1991; Thorndyke \& Hayes-Roth, 1982).

Many different techniques have been used to elicit spatial products (externalized representation of a respondent's knowledge). For example, respondents may be asked to draw a sketch map of an area (Blades, 1990), locate points on a base map (Buttenfield, 1986), estimate the distance (Day, 1976) or direction (Kirasic, Allen, \& Siegel, 1984) to a series of other locations, recognize features on an aerial photograph (Matthews, 1984), retrace their path along a route (Passini \& Proulx, 1988), verbally describe a route or an area (Vanetti \& Allen, 1988), or build a scale model that represents an area (Hart, 1979). It is apparent that although groups of individuals 
share common knowledge, there are individual idiosyncratic differences in spatial products (Lloyd, 1989). However, we are unsure as to whether individual differences are the result of differences in knowledge, the ability to cope with the task set or cognitive strategies of thought. Foley and Cohen (1984) argued that cognitive mapping abilities differ between individuals because of qualitatively different encodings and representations. Individuals thus use different types of strategies to encode and decode their knowledge and, as a result, differ in their answers to cognitive mapping tasks. They suggested that different strategies of thought or decoding will lead to radically different results, even when the knowledge base is identical. Miller (1985) similarly argued that individuals differ in their cognitive styles of thought.

In addition, several studies have noted that different tests produce varying results (Bryant, 1984; Cadwallader, 1979; Howard, Chase, \& Rothman, 1973; Magana, Evans, \& Romney, 1981; Matthews, 1984; Montello, 1991) and yet the specific reasons for these variances are unknown. However, there has not been an attempt to discover the strategies of thought used in performing tasks designed to measure aspects of cognitive map knowledge; how many strategies are used; the relationships between different strategies of thought; and if different strategies of thought lead to different results within and across tests. These are important questions that raise issues relating to validity and integrity (Kitchin, in press). In this article, these questions are preliminarily examined and some of the results from an exploratory phenomenological investigation of spatial thought are reported. Spatial thought is a term first used by Liben (1981) to describe strategies used to complete cognitive mapping tasks. The study focuses on the strategies of spatial thought used to complete tasks designed to measure configurational knowledge.

Traditionally, cognitive map knowledge has been divided into a series of three knowledge structures through which an individual progresses. Liben (1981) described declarative knowledge as the mental database of specific spatial features. Declarative knowledge is generally regarded as landmark 
knowledge, although it can include linear features, such as roads, or areal features, such as parks (Golledge, 1993). Procedural knowledge consists of the rules used to synthesize declarative knowledge into information that can be used to facilitate an action. For the purpose of cognitive mapping, these rules are essentially wayfinding knowledge that direct movement between places, an example of which would be the transformation of path elements into a navigable route (Thorndyke, 1983). This transformation, however, does not include the ability to make inferences about routes never experienced. Configurational knowledge incorporates information, such as angles, directions, orientation, location, and distance apart of places (Golledge, Gale, \& Richardson, 1987), so the possessor has knowledge of the associations between, and the relative positions of, places; these form a comprehensive spatial knowledge system (Golledge, 1992).

\section{METHODOLOGY}

\section{BACKGROUND}

The study aimed to use a qualitative methodology to determine how people completed the cognitive mapping tests, by observing the participants while they completed the tests and asking them questions relating to how they were trying to complete the task. Such an approach is described as phenomenological as it focuses on the relationship between the respondents of the study (undergraduates) and the object of the research (their spatial thought) (Gerber \& Kwan, 1994). Phenomenology "requires us to reflect on our own consciousness of things and on our own experience [to] come to a deeper understanding of ourselves" (Jackson \& Smith, 1984, p. 27). In the context of this article, respondents were required to reflectively analyze their thoughts and experiences while undertaking a particular task that was to be analyzed empirically. In a sense, the construct validity (whether the test was measuring what it 
is supposed to without introducing bias; Coolican, 1990) of the tests was being examined.

A phenomenological approach has three principal advantages, allowing the investigator to learn about and gauge the extent of the respondent's configurational knowledge; to understand how the respondent is attempting to complete the task; and to attempt to discover the form and structure of respondents' knowledge. Gerber and Kwan (1994) in a unique study examined how 12-year-old adolescents learned a neighborhood using a map by adopting a phenomenological approach. However, phenomenological work concerning how respondents perform and think on spatially based tasks remains rare, although a little research has used verbal estimates or descriptions to understand cognitive map knowledge. For example, Taylor and Tversky (1992) asked their respondents to describe their cognitive maps verbally to try to discover the hierarchy or anchors of such knowledge. Places that were mentioned either first, or more frequently, were assumed to have more significance, acting as anchors for other knowledge. Vanetti and Allen (1988) compared the production and comprehension of route directions between four groups of respondents who differed in their verbal and spatial ability. No differences were found between the groups in regard to the respondents' abilities to comprehend the routes directions. However, spatial ability was found to be important in the production of efficient route directions and verbal ability tended to conform the communication of route knowledge to certain linguistic conventions based on the number of descriptors and delimiters.

Landau and Jackendoff (1993, p. 217) have argued that any "aspect of space that can be expressed as language must also be present in non-linguistic spatial representations. Simply put, whatever we can talk about we can also represent." The argument forwarded here is that to an (unknown) extent what we can represent we can verbally describe: there is a correspondence between talking about spatial representations and thinking about them. As such, verbal abilities can be used to effectively communicate spatial knowledge and ability (Vanetti \& Allen, 1988). Some have argued that verbal reporting of cogni- 
tive processing needs to be treated with care, suggesting that although individuals have direct access to their knowledge, such control does not exist for their mental processes (Nesbitt \& Wilson, 1977). However, Ericsson and Simon (1980, p. 247), on the basis of an extensive literature review, argued that verbal reports are "a valuable and thoroughly reliable source of information about cognitive processes" when elicited with care and interpreted within the context they were collected.

\section{RESPONDENTS}

The respondents for the investigation consisted of 40 , volunteer, first-year geography undergraduates resident at the University of Wales Swansea. The respondents were recruited from geography practical classes. All had been resident in Swansea for approximately one term (9-10 weeks) and were aged between 18 and 29. None of the respondents were told about the nature of the experiment that they had volunteered to undertake and had not been taught concepts relating to cognitive mapping. All had similar geographic training and access to maps and geographic details of the Swansea area. As part of their first-year practical packs, each had received an Ordnance Survey Landranger (1:50000) map of the Swansea and Gower area. The practicals for the first term had provided them with map design skills, taught them how to understand maps, and had given them a broad knowledge of the South Wales area; practicals tended to be at the West Glamorgan (county) and South Wales scale rather than the Swansea (city) scale. All had been on a department geohistoric tour of the city and its surrounding area in the second week of arrival. It is noted that these respondents had access to information and skills not normally available to most students.

\section{THE TESTS}

Respondents were divided into two groups of 10 females and 10 males, who each completed two of four tests designed to measure their configurational knowledge of the Swansea area. 
The first group completed a sketch map and a cloze procedure test (Figure 1). Sketch mapping, despite well-documented faults relating to scaling and graphicacy (see Blades, 1990), remains a popular method of collecting configurational knowledge. There are five basic variations (Kitchin, 1995). The basic sketch map technique is designed to obtain from the sketch mapper a freely drawn and solicited sketch map that has been minimally defined by the researcher (e.g., Jacobson, 1992). The respondent is given a blank piece of paper and asked to map a given environment. The normal sketch mapping technique imposes more constraints on the respondent than the basic approach (e.g., Saarinen, MacCabe, \& Morehouse, 1988). The researcher is often interested in more specific features and will word the instructions appropriately to obtain the required data. In cued sketch mapping data collection, the respondent is given a portion of the map and asked to complete specific features (e.g., Pearce, 1981). The longitudinal sketch map technique allows the researcher to study how the sketch map evolves. The instruction set is similar to the normal procedure, but it requires the respondent to provide the sketch map on layers of carbon or tracing paper. After certain time periods, the sheets of paper are turned over and the respondent continues to draw (e.g., Humphreys, 1990). Wood and Beck (1976) and Beck and Wood (1976) have argued that teaching respondents a sketch map language produces maps that are not compromised by graphic ability or a lack of mapping knowledge. They developed a sketch map language called "Environmental $A$ " for use by school children. The sketch map test used in the present study is of a normal design. The cloze procedure test is a spatial completion test. Traditionally, the respondent fills in the missing space, and an aspatial example would be, "A dog barks but a cow _ ? Robinson (1974) and Boyle and Robinson (1979) extended this exercise spatially. Abase map is covered in a grid, and the information contained in some of the squares is deleted. Respondents are then asked to identify particular elements in these blank squares with the aid of contextual information retained in the remaining open squares. 


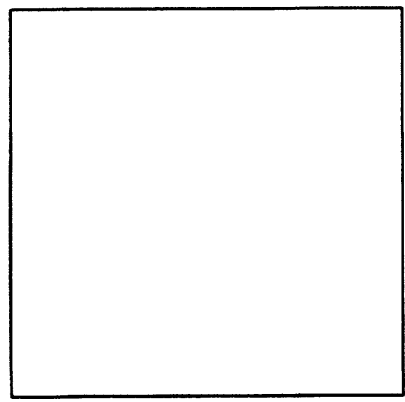

Sketch map test: Draw a map of Swansea inside the box. Include any places or landmarks that you think you know the location of.

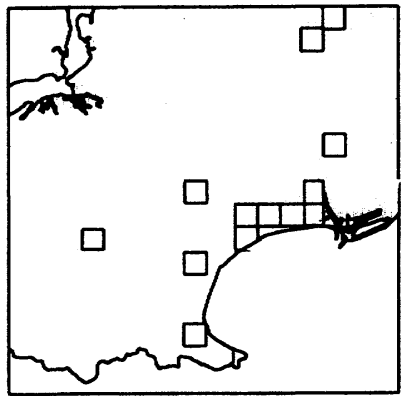

Cloze procedure test: Write in the blank boxes the number of the place or landmark from the fifteen listed below that has been removed (a list was provided to each respondent).

Figure 1: The Sketch Map and Cloze Procedure Tests

The second group completed a projective convergence and an orientation specification test (Figure 2). The projective convergence (or resection) method uses distance and direction estimates to work out the coordinates of locations. Typically, respondents estimate the distance and direction to unseen places from three or more locations. The resulting vectors can be drawn and where the lines end a triangle of error can be drawn whose mean center is taken as the cognitive location of a place (Figure 3B). Hardwick, McIntyre, and Pick (1976) originally developed the method in a study where respondents first familiarized themselves with four locations within a library. Screens were then erected and respondents asked to estimate the direction by pointing a sighting tube in the direction of the four unseen locations. By calculating where the lines inter- 
1. Singleton Hospital to Swansea Railway Station.

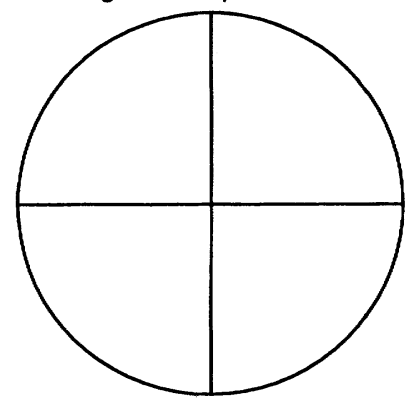

Projective convergence test: The centre of the compass represents the first place. Mark with a line the directionto the second place. The length of the line will represent the distance from the first to the second place where the compass radius is equal to the striaght line distance between Mumbles Pier and Morriston Hospital which is 8.1 miles.

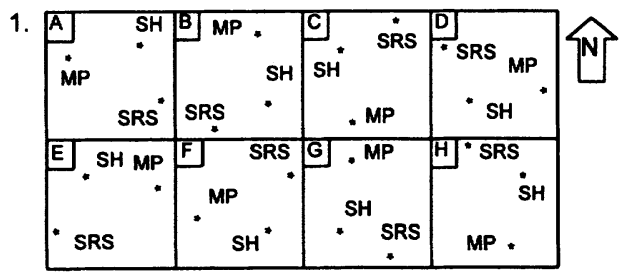

Square is the correct orientation.
Orientation specification test: Complete the booklet detailing which box containsthe correctly orientated configuration.

MP Mumbles pier SH Singleton hospital SRS Swansea rail station

Figure 2: The Projective Convergence and Orientation Specification Tests

sected, the triangle of error could be found and a cognitive location could be calculated (Figure 3A). Kirasic, Siegel, and Allen (1981) first used the distance/direction method (Figure 3B) to study 48 students' memory of locations on a university campus, using a direct magnitude method for eliciting distances. In a second experiment (Kirasic et al., 1984), they devised a method whereby distance and direction were recorded simultaneously with respondents drawing a line that represented both. Orientation specification is a form of recognition test. Recognition methods collect configurational knowledge data by providing the respondents with a representation of an environment and asking them to correctly identify features and configurations. For example, Evans, Fellows, Zorn, and Doty (1980) asked respondents to identify four out of eight floor 


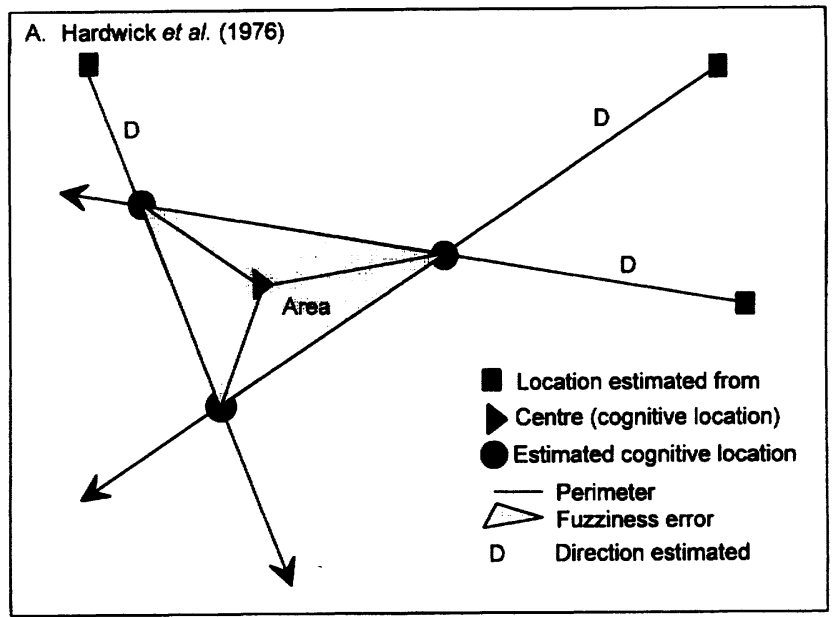

B. Kirasic et al. (1981)

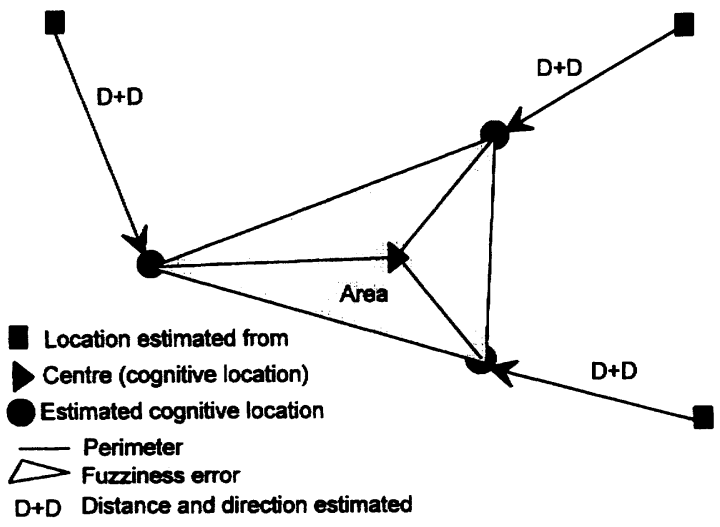

Figure 3: Two Methods of Projective Convergence Resection

plans they had just walked through. Evans and Pedzek (1980) gave respondents a set of triad configurations, half of which had the correct configuration and half an incorrect configuration. These configurations were either nonrotated or rotated by 60 , 
120 , or 180 degrees. Respondents were shown the triads one at a time and asked to say which configuration had the places corrected located relative to each other, despite the rotation, and the reaction times were noted.

These four tests were chosen because they differ in natureall requiring the respondents to complete tasks that varied in the information provided and the information requested, but which have all been used in the past to measure configurational knowledge. All respondents completed the tests while facing north in a room where only the front of the building opposite was visible through the window. Respondents completed the tests while undertaking semi-think-aloud protocol interviews. Thinkaloud protocols were developed by Newell and Simon (1972) and consist of the respondents describing their actions or thoughts while performing a task; it is literally a running commentary. It aims to discover the knowledge and cognitive processes used in problem solving, but it is not without criticisms. First, although respondents are meant to be giving a description of their experience while thinking, they often fall into the habit of just giving expression to their thoughts. This gives information that is independent of, or irrelevant to, the actual mechanisms of problem solving. A second criticism concerns an "incomplete argument," whereby respondents fail to verbalize and track the actual path of activity. Third, verbal reports may be reporting an activity that is taking place in parallel with, but independently of, the actual thought processes providing no reliable information about the required cognitive processes. Last, verbalization could alter thought processes and invalidate the results.

To try to reduce these problems (and a further problem noted in a pilot study that respondents found it difficult to talk and perform the task at the same time), a semi-think-aloud/selfreport protocol procedure was adopted whereby respondents were encouraged to perform a think-aloud protocol, but were also asked questions while performing the tasks, requiring them to describe their thoughts and actions. The respondents received prompts for information if they fell silent for more than 10 seconds. The prompts were open-ended and designed not to aid task completion, being more general primers such as 
TABLE 1

Strategy Structural Frames and Codes for Table 2 and Table 3

Code Description

Common strategies

C1 Imagining or constructing various types of maps.

C2 Referring to the coastline.

C3 Imagining the route or traveling between two locations.

C4 Using travel time to work out the separation between locations.

C5 Imagining standing at a location and "looking" in the direction of another location.

C6 Imagining looking down vertically or obliquely.

C7 Working out where places are in relation to the current location.

C8 Just know-propositional coding.

Common task strategies

CT1 Elimination.

CT2 Logical deduction.

CT3 Draw a map.

CT4 Where the sun sets.

CT5 Look back at former answers.

Task-specific strategies

Cloze procedure

T1 Look at square, decide what is located there and check to see if on list.

T2 Look at list to see if recognize any, then go to the map to see if a square is free where you think it is.

T3 Swap strategy.

Projective convergence

T4 Work out the direction between two locations by working out the direction from each place to a third place (e.g., for A to B work out direction from A to $C$, and then $C$ to $B$ ).

T5 Imagine flying as a crow would between two locations.

T6 Instead of working out the route from $A$ to $B$, work out $B$ to $A$.

17 Draw a route map across the projective convergence circle.

T8 Imagining a map directly in front of yourself and lining a pencil up between the two locations and moving the pencil down across the circle.

Orientation specification

T9 Work out how it should look like then scan all the squares for one that fits.

T10 Work out how it should look like then work systematically through the squares until one fits, choose that and ignore the rest of the squares.

T11 Draw the coastline onto the configurations.

Quantitative results

$P \quad$ Number of paths.

E Number of edges.

D Number of districts.

N Number of nodes.

L Number of landmark.

CZ Cloze procedure completion score.

PR Projective convergence bidimensional regression $r^{2}$ value.

OS Orientation specification completion score. 
TABLE 1 (Continued)

\begin{tabular}{cl}
\hline Code & \multicolumn{1}{c}{ Description } \\
\hline Table codes \\
s & Sketch map \\
c & Cloze procedure \\
p & Projective convergence \\
o & Orientation specification \\
x & Category response best fits into. \\
$\$$ & Swapped strategy only when completed stuck using original strategy. \\
\hline
\end{tabular}

"what are you doing/thinking now?" The respondents were told that they were to complete the two tests while articulating their thoughts and actions and that they would also be asked questions such as "how do you know that place is there?" They were given no details about how to complete the tests with specific instructions omitted, for example, "you may look back at previous answers." The interviewer also gained evidence by watching the respondents' reactions. This was immediately followed by a semistructured debriefing interview that aimed to validate the protocol analysis, and to allow further discovery concerning the nature of the tests. Respondents were asked questions relating to which tests they preferred, found the easiest, best represented their knowledge and why, and to recommend alternative data collection methods that may have been more successful in eliciting their configurational knowledge of the Swansea area.

\section{ANALYSIS}

The interviews were taped and analyzed first through direct transcription and then using a series of structural frames that acted as a set of filters. Structural frames are categorizers defined by the information provided by the respondents. Each new frame was constructed by the author, so that each new strategy articulated by the respondents became the basis of a new frame. Table 1 details the structural frames identified and the codes for Table 2 and Table 3. Three sets of frames were 
discovered. Common strategies of spatial thought are general strategies of accessing and manipulating cognitive map knowledge to complete the task set (e.g., imagining a seen map to complete a task). Common task strategies are general problemsolving strategies relating to specific problems incurred when attempting a task that are applicable in a number of situations (e.g., using an elimination strategy to narrow choices). Taskspecific strategies are problem-solving strategies specific to just one test and are used to overcome unique circumstances (e.g., working out the distance and direction between places $A$ and $B$, and $B$ and $C$ to obtain $A$ to $C$ ). Table 2 and Table 3 display the structural frames (each strategy) used by each individual (each row) on each of the two tests they completed plus the quantitative results for each test. The female respondents are identified by an $F^{\star}$ identifier (e.g., $F 1$ ) and the males by an $M^{\star}$ identifier (e.g., M1). It must be noted that strategies indicated in the tables are only those articulated by the respondents and that some respondents might have been using strategies that have not been recorded. This is almost certainly the case and it was noted during the interviews that some participants were clearly using other strategies that they were then failing to articulate to the interviewer. As a result, the findings presented in this article only represent the information as transcribed. Nonetheless, the findings are of worth as they allow an insight into the strategies used in spatial thought and their effectiveness.

All four tests were also analyzed using traditional quantitative techniques, so that the relationship between externalized knowledge and the reported strategies of spatial thought could be examined. The sketch map data were analyzed using Lynch's (1960) content analysis (Table 4), with each map's elements tallied into the appropriate category. The cloze procedure test was analyzed by constructing a completion score that represented how well an individual did in assigning the places to the boxes. A score of 100 means all of the boxes had correct locations assigned to them, and a value of 0 that all of the boxes had an incorrect place assigned to them. The projective convergence test (Kirasic et al., 1984) was analyzed by converting the distance and direction estimates into a set of coordinates 
using a resection methodology (Figure 3B). These coordinates were then bidimensionally regressed onto the real world coordinates using the CMAP package (Kitchin, 1994b). Bidimensional regression (Tobler, 1965, 1976) is a two-dimensional equivalent of ordinary least squares linear regression and allows two sets of coordinates to be quantitatively compared with the $r^{2}$ value denoting the amount of association. The orientation specification test was analyzed by constructing a completion score representing the number of configurations correctly identified. The completion score ranges between 0 and 100 , with a value of 0 indicating that the respondent had not correctly identified any configuration and a value of 100 indicating that all the configurations had been correctly identified.

\section{RESULTS AND DISCUSSION}

\section{EXPLORING COMMON STRATEGIES OF SPATIAL THOUGHT}

Common strategies of spatial thought refer to ways of thinking that could be applied to most cognitive mapping tests, and are not constrained by the specific task demands of a particular test. Eight such strategies of thought were encountered in conducting the semi-think-aloud protocols that varied greatly in their popularity. One of the most popular strategies employed by individuals to attempt the tasks was to use map imagery. This typically took one of two forms. First, some respondents tried to remember a map that they had seen, typically an ordnance survey map, in its entirety. It seems that they had stored this map as a complete image or as component images that they then reconstructed to perform the task. Second, some tried to construct maps that contained a minimal structure or only contained features necessary to complete the task. It was implied and sometimes explicitly stated that these maps were constructed for the purpose of the task. Some respondents claimed that they swapped between their constructed minimal map and remembering an ordnance-survey-based map on 


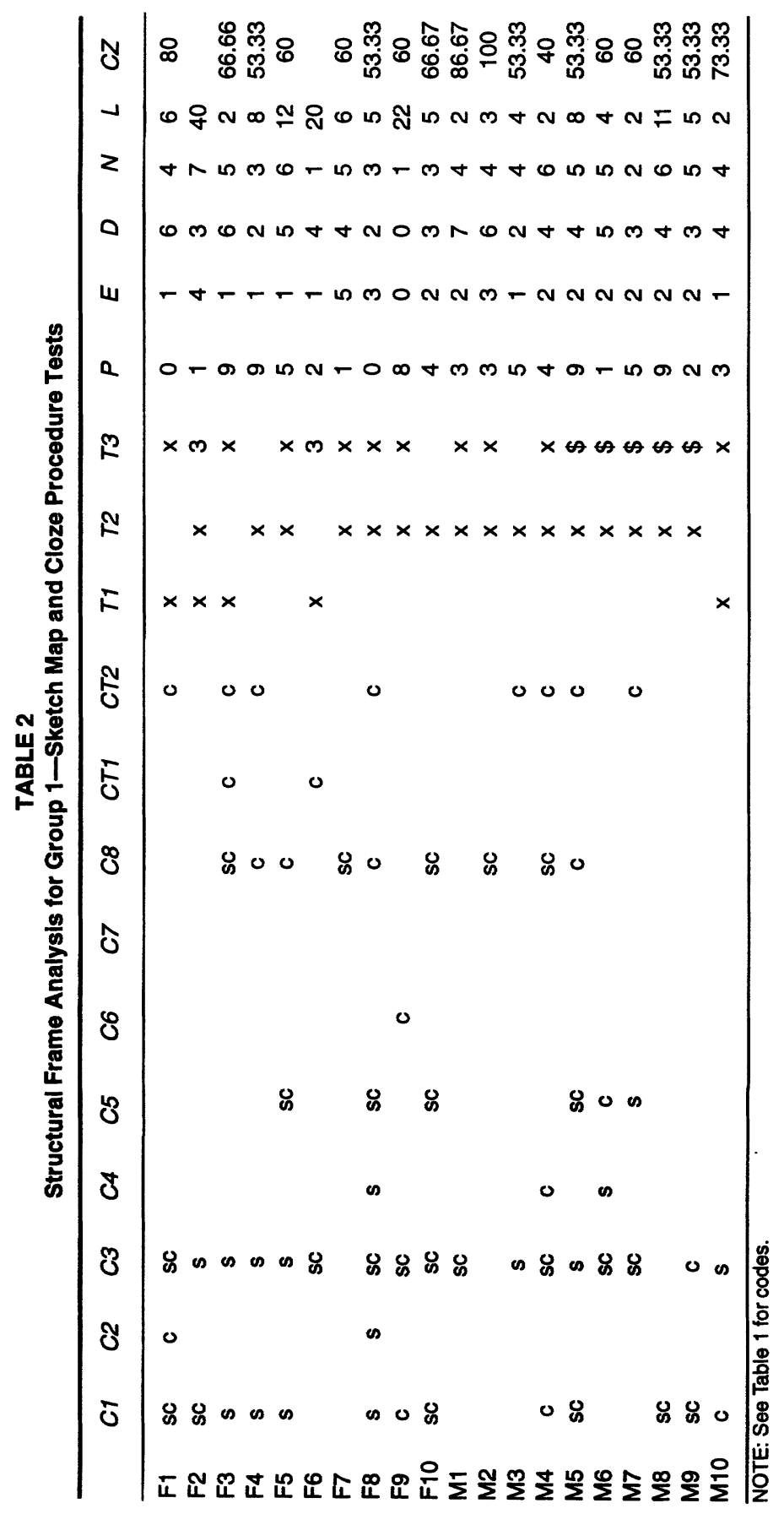




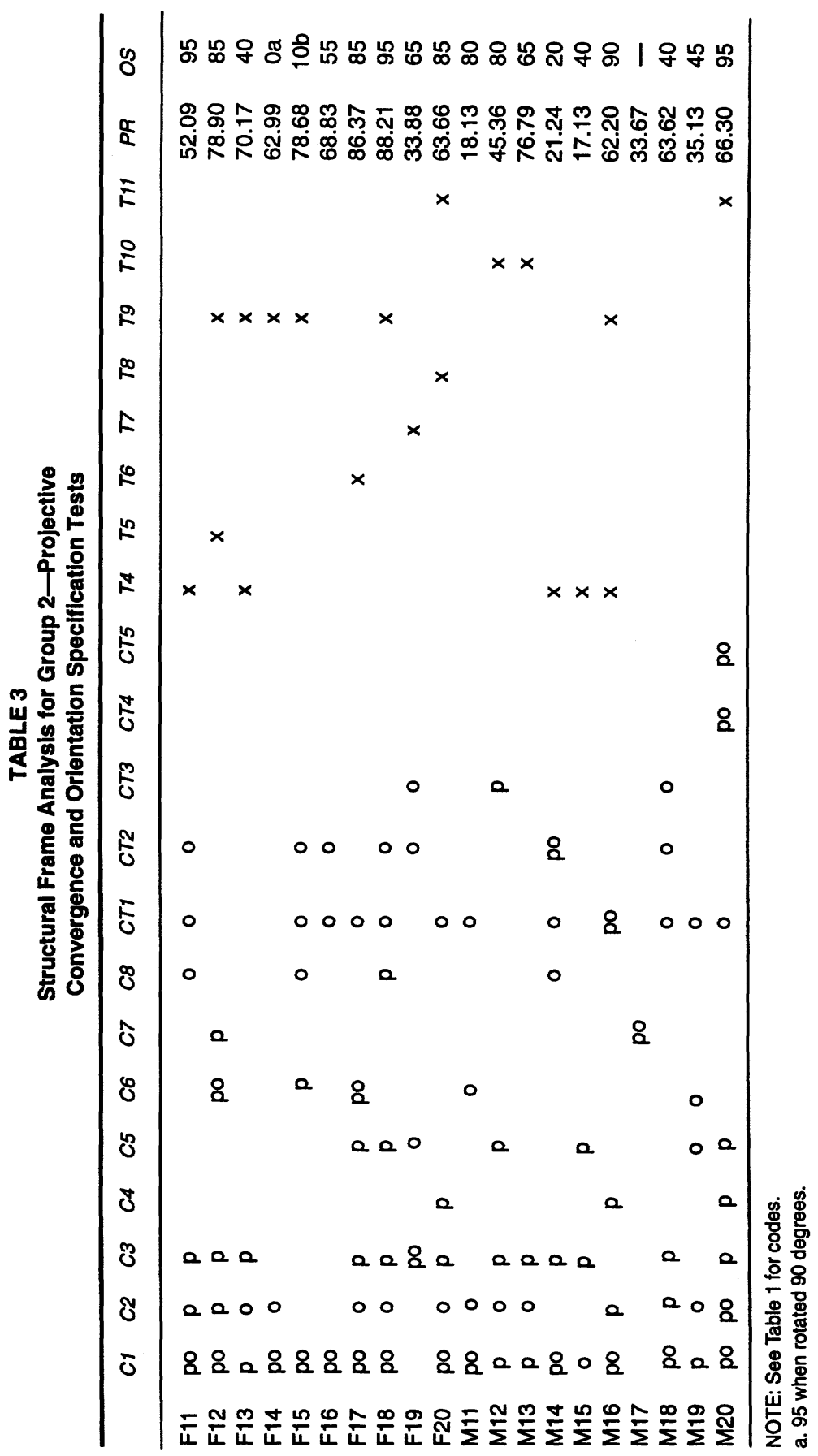


TABLE 4

Lynch's (1960) Content Classification

\begin{tabular}{ll}
\hline Category & \multicolumn{1}{c}{ Verbal Description } \\
\hline Paths & $\begin{array}{c}\text { Paths are the channels along which sketch mapper moves. They may } \\
\text { include streets, walkways, railways. } \\
\text { Edges are the linear elements not considered as paths by the sketch } \\
\text { mapper. They are the boundaries between two phases, linear breaks in } \\
\text { continuity such as shores or walls. } \\
\text { Districts are the medium-to-large scale sections to the city, conceived as } \\
\text { having a two-dimensional extent, which the observer mentally enters } \\
\text { inside of, and which have some common identifiable character. } \\
\text { Districts } \\
\text { Nodes are points, the strategic spots in the city into which an observer } \\
\text { can enter, and which are the intensive foci to and from which he is } \\
\text { traveling. They may be primarily junctions, places of a break in } \\
\text { transportation, a crossing or convergence of paths. } \\
\text { Landmarks are another type of point-reference, but in this case the } \\
\text { observer does not enter within them, they are external. They are usually } \\
\text { a rather simply defined physical object: building, sign, store or mountain. }\end{array}$ \\
Landmark &
\end{tabular}

questions that they found more difficult to answer, but in other cases the constructed map would be a combination of remembered and constructed map, intertwining elements from both. For example, respondent M8 could remember the road network from his road atlas and into this he would add locations that he had visited. There were, however, some problems encountered by respondents attempting to use a mapping strategy. Some found that they could not successfully build a map because of incomplete knowledge. Respondent F20 was conscious that her own constructed map was not in fact aligned along a north-south axis and as a result she was having to rotate her answers to fit the options provided in the orientation exercise. However, she found such a rotation mentally difficult and as a result struggled to complete the test.

Throughout the interviews, it became clear that the coastline is the dominant anchor for residents in Swansea. This anchor is so dominant in fact that some respondents, when explaining how they had completed tasks, suggested that they had worked out locations solely in relation to the coastline. Their base maps 
were so minimal that this was the only feature it contained and the responses were plotted in relation to it before being transposed onto the spatial product. This strategy was used to some extent across all of the tests, but the coastline's salience was best illustrated by respondents completing the orientation test. Two respondents completed this task by drawing the coastline onto the configurations to see which was correct, using this cue to check their answers. Others claimed to mentally draw such a line over the configurations.

Another of the most popular strategies of spatial thought is imagining traveling along a route. Typically, respondents expressed the notion of imagining a journey between the two places. This expression took two different forms. Most respondents declared a route strategy best described as a replaying of a route traveled. Instead of imagining a journey between two locations, some respondents constructed a minimal map that only contained the route between two places. In the main, these types of route maps were created not by remembering a map but by constructing them, converting route knowledge into configurational knowledge. Respondents would imagine traveling along a route and then simultaneously transpose this knowledge onto a mental base map of the area that would then be used to answer the question. One respondent, F1, explained that she used the two methods of traveling a route and using a map strategy to validate the results from one with the other to provide a composite answer. Like the map strategy, some respondents experienced difficulty using the route strategy. Respondent $\mathrm{F} 1$ used this combination strategy because she did not possess enough map knowledge for a particular question. Equally, she found the route strategy alone too taxing. The route traveling strategy proved to be a popular choice because it closely corresponds to the real-world interaction that the respondents undertake. It was not reliant on memorizing details from a map or an abstract concept of thought, but on information actually experienced at first hand.

A few respondents used their knowledge of travel times to work out the separation or distance between places. This was 
exclusively based on firsthand walking or cycling experience, where the amount of time taken to traverse between the two places was mentally converted into distances using an individual conversion standard. For example, one might convert half an hour's walk into 2 miles; someone else into 1-1/2 miles. Approximately a third of all respondents used the strategy of imagining being at a location and looking in the direction of another location, at some point during one of the tests. Typically, respondents would describe standing at a location, in this case mostly Mumbles Pier, and looking across the space in front of them to judge either distances, directions, or where two places were in relation to each other. The strategy is fairly limited in that it needs an area that allows viewing of such panoramas, but proved to be an effective strategy for judging the spatial relationships of places located along the coastline. Some respondents constructed answers using abstract strategies that were based on an amalgamation of real-life route knowledge and that gleamed from a map, so that they tried to solve the task using a strategy never experienced. One of the ways they attempted this was imagining looking down onto Swansea as if they had a bird's-eye view from either vertically above or at an oblique angle.

One respondent worked out the positions of other locations solely in relation to where he was currently located. So, for example, a respondent might work out where Mumbles Pier is by thinking "if I am here then it must be over there." Although only one respondent used this strategy, it is one that could be potentially used on all of the tests. Respondent M17 was unusual in that he only used this strategy on both of the tests he performed. As a consequence of this egocentric knowledge, respondent $\mathrm{M} 17$ had to orient the test to the direction he thought north was. This was at 90 degrees to its true direction and so he had to rotate the test 90 degrees to the right to be able to attempt the tasks. Even when rotated, he had so much difficulty with the orientation specification test that it was eventually abandoned. Although respondent $\mathrm{M} 17$ had problems using this strategy, it has the potential to be a successful strategy if a bearing is known before the test starts. If the test is done when 
the respondent is disoriented, then it will inevitably lead to results that do not reflect the respondents true knowledge.

A certain number of the respondents claimed to "just know" the answer to a task. They did not need to employ any strategy of thought because they automatically knew where certain places were in relation to each other. Many implied some type of propositional coding, whereby information was coded in the form "along the sea front then in a bit" with respondents knowing the directions and distances instinctively. Propositional statements such as "near to" or "left of" were common with no references made to any imagery. It is difficult to validate this strategy in that the respondents could in fact be describing the answer resulting from another strategy. For example, respondents may know that the hospital is next to the university because they had just consulted a map image strategy. However, the definite impression given by respondents was one of "just knowing," and some when asked whether they did just know or whether they had used another strategy such as thinking of a map most replied that they "just knew." Propositional coding seems a plausible way of encoding configurational knowledge, as long as the spatial relationships are encoded, and other researchers such as Foos (1980) have found that propositional data can be encoded and processed into cognitive map knowledge.

\section{TASK-SPECIFIC STRATEGIES OF SPATIAL THOUGHT}

Task-specific strategies of spatial thought refer to strategies that relate to solving the tests rather than the decoding of information. They can be divided into two main categories. First, common task strategies are those that can be applied across different tests where certain tasks are similar or identical. Second, specific task strategies refer to those strategies that are unique to those tests. There were five basic common task strategies encountered across the four tests that varied in popularity. Each strategy was used on at least two of the tests.

The strategy of elimination was commonly employed on the orientation specification test and once on the cloze procedure 
test. The orientation test, because it provided the solution and just required the respondent to say which was the correct configuration, allowed the easy adoption of the elimination strategy. With this strategy, respondents could arrive at an answer, not by first thinking of the answer and then looking for the correct response, but rather by eliminating all of the configurations they knew were incorrect to leave one answer or narrowed choice. Typically, respondents employed this strategy when they were not sure of the correct answer or could not find an answer that matched their own thoughts. By eliminating all of the answers they knew were definitely incorrect, they often narrowed down the choice to two, or less commonly three, remaining options. At this point, they would then change strategy to try to eliminate the remaining square(s).

Some respondents, when faced with the problem of not knowing where a place was, would use a system of logical deduction, whereby they would use other information to obtain a answer. For example, on the cloze procedure tests, most respondents placed the Swansea-Cork ferry terminal in the correct square, not because they knew where it was, but because they knew it had to be on the coast. Similarly, Swansea airport was often located on the outskirts of Swansea because that seemed a sensible location for an airport to be sited given the problems of noise pollution and the need for flat ground. On the orientation test, some participants would determine the answer on the basis of knowing where just one or two places were sited. For example, if two of the locations were in the correct location, they would assume that the third was there also and choose that answer. The use of logical deduction was often accompanied by the use of elimination.

Three of the respondents found drawing a map a good strategy for trying to answer the projective convergence and orientation tests. By externally expressing their knowledge, they could then either directly transpose that knowledge onto an answer sheet by visual perception for the projective convergence or match their answer to the answers provided for the orientation test rather than cognizing the information for each section of the tests. Only one respondent used where the sun 
sets in determining the answers, although it has the potential to be a successful strategy. Again, the same respondent, M20, was the only respondent to look back at his previous answers to aid the process of completing the current task. All of the other respondents tried each part of the test independently without referring back to their previous answers. M20 also came up with the ingenious method of combining two answers. On two previous tasks, he had been asked to estimate the distance and direction from Singleton Hospital to Swansea Rail Station and Mumbles Pier. When asked to estimate the distance and direction to Mumbles Pier from Swansea Rail Station, he redrew the two previous estimates as if Singleton Hospital was meant to be the central point and then translated the two estimates until Swansea Rail Station became the central point, thus ensuring that his answer conformed exactly to the previous answers. However, once he had done this, he changed his mind and moved Mumbles Pier to a more westerly position without altering the previous answers; his preconceptions were overriding his logic.

There were two main task-specific strategies adopted by respondents completing the cloze procedure test. The first and least popular was to look at the map provided, decide which location could be sited in a particular square, and then go to the list to see if that location was listed. The second, involved scanning the list for places that were familiar and the location known, and then look at the map to see if a blank square existed in the site spatial thought had determined it was located. Most respondents $(80 \%)$ initially adopted the second strategy with many swapping to the first strategy while completing the test. A number of the males $(50 \%)$, however, left the swapping of strategy until the point where they became stuck or realized that they had made a number of errors that needed altering. It seems that they had adopted a strategy that they thought was successful and as a result continued with that strategy until it failed. The females, however, took much more notice of how their answers were shaping and corrected their mistakes at a much earlier stage by swapping strategies. The late swapping of tactics by the males did not lead, however, to them underachieving in 
relation to the females (two-sample $t$ test, $t=0.95, p=.36$ ); it seems that the fact that they did change and alter their answers led to comparable results. Only three respondents did not alter their strategy at all and these also gained results, once the guesses were removed, comparable to those who did swap strategy. From this it can be concluded that the choice of task-specific strategy on the cloze procedure test had little effect and did not lead to different results.

There were five projective convergence specific task strategies but only the "via another location" proved to be popular. Using this strategy, respondents would work out the distance and direction to a location by mentally "going via" another location. For example, they might work out the distance and direction from $A$ to $B$ and $B$ to $C$ to determine $A$ to $C$. This is similar to the strategy used by respondent M20 when looking back at previous answers. Here, however, the calculation was carried out mentally and did not involve looking at past answers; each particular task was completed independently. In particular, two questions were tackled using this strategy. The distance and direction from Swansea Rail Station to Mumbles Pier and Swansea Rail Station to Hendrefoilan were both calculated via the University or Singleton Hospital. These pairs were two of the most separated places and it may be the case that respondents were dividing them into more manageable chunks. Others said that they used this strategy when they were not familiar with the direct route between the two.

The other strategies used for the projective convergence test were only used by single female individuals. Respondent F12 described the abstract strategy of flying as the crow flies between the two locations. F17 worked out some of the distances and direction by imagining traveling between $B$ to $A$ rather than $A$ to $B$, a task she found easier. Respondent F19 drew a map of the route across the circles of those she found difficult and then connected up the end points. This was a tactic that seemed to be used by many respondents who traced the route in the air above the circle, but when asked about what they were doing did not articulate about this gesturing. F20 imagined a map directly in front of her, held her pencil up between the two 
locations on this imagery map, and then carefully lowered the pencil to the paper to draw on the direction.

There were three basic task-specific strategies used on the orientation test. Two worked on the principle that you decide how the configuration should look and then try to find a square that contained that configuration. They differed, however, in the response to finding the correct configuration. Respondents could then either scan the rest to see if they had made the right choice or they could stop and choose that answer, ignoring the rest. Only one respondent chose the second strategy, feeling confident that he made the right choices. The third strategy adopted by two of the respondents was to draw the coastline onto the configurations to check that the answer they had decided on was correct. It seems that they needed to physically transpose their main spatial anchor to give the configurations context.

\section{THE RELATIONSHIP BETWEEN STRATEGY AND RESULTS}

It is clear from the discussion so far that a range of common, common task, and task-specific strategies of spatial thought was adopted by respondents trying to complete the four cognitive mapping tasks. However, from this analysis of the transcripts, it is difficult to determine if there is a relationship between the common strategies of spatial thought adopted and the quantitative analysis. To determine if such a relationship exists, a number of ANOVA tests were calculated. The ANOVA tests indicated that the adoption of certain strategies of thought did not lead to more accurate results on any of the tests (sketch map test, $f=0.51, p=.726$; cloze procedure test, $f=0.04, p=$ .987; projective convergence test, $f=0.55, p=.791$; orientation specification test, $f=0.40, p=.804$ ). In addition, the number of strategies adopted by respondents do not differ across the tests, that is, certain tests do not increase the likelihood of adopting more strategies of thought (one-sample chi-square test $\chi^{2}=2.97, p<.95$ ). It is clear from the ANOVA tests that respondents adopt a range of common strategies, but that these strategies are equally likely to provide a solution to the 
TABLE 5

Investigating the Role of Strategy and Metacognition on the Sketch Map Test

\begin{tabular}{|c|c|c|c|c|c|c|c|c|c|c|c|}
\hline Respondents & $\begin{array}{l}\text { N-Degree } \\
\text { Places } \\
\text { Located }\end{array}$ & $\begin{array}{c}\text { N-Degree } \\
\text { of Common } \\
\text { Strategies } \\
\text { (+ Other) }\end{array}$ & C1 & C2 & C3 & C4 & C5 & C6 & $C 7$ & $C 8$ & $\begin{array}{c}\text { Meta- } \\
\text { cognition }\end{array}$ \\
\hline M8 & 32 & 1 & $x$ & & & & & & & & + \\
\hline F9 & 31 & 1 & & & $x$ & & & & & & + \\
\hline F5 & 29 & 3 & $x$ & & $x$ & & $x$ & & & & $=$ \\
\hline M5 & 28 & 3 & $x$ & & $x$ & & $x$ & & & & - \\
\hline F3 & 23 & 3 & $x$ & & $x$ & & & & & $x$ & - \\
\hline F4 & 23 & 2 & $x$ & & $x$ & & & & & & + \\
\hline$F 7$ & 21 & 1 & & & & & & & & $x$ & $=$ \\
\hline F2 & 18 & 2 & $x$ & & $x$ & & & & & & $=$ \\
\hline M1 & 18 & 1 & & & $x$ & & & & & & $=$ \\
\hline M4 & 18 & 2 & & & $x$ & & & & & $x$ & $=$ \\
\hline F1 & 17 & 2 & $x$ & & $x$ & & & & & & $=$ \\
\hline $\mathrm{F} 10$ & 17 & 4 & $x$ & & $x$ & & $x$ & & & $x$ & - \\
\hline M2 & 17 & 1 & & & & & & & & $x$ & + \\
\hline M6 & 17 & 2 & & & $x$ & $x$ & & & & & - \\
\hline M9 & 17 & 2 & $x$ & & & & & & & $x$ & $=$ \\
\hline M3 & 16 & 1 & & & $x$ & & & & & & $=$ \\
\hline M10 & 14 & 1 & $x$ & & & & & & & & $=$ \\
\hline M7 & 14 & 2 & & & $x$ & & $x$ & & & & $=$ \\
\hline F8 & 13 & 5 & $x$ & $x$ & $x$ & $x$ & $x$ & & & & - \\
\hline F6 & 11 & 1 & & & $x$ & & & & & & - \\
\hline
\end{tabular}

NOTE: + Respondent thought he/she had a good knowledge of Swansea; = respondent thought he/she had a reasonable knowledge of Swansea; - respondent thought he/she had a poor knowledge of Swansea.

task set. These results must be treated with a certain amount of caution because it is difficult to determine the relationship between strategy and results as there are other factors that are difficult to account for, such as the amount of underlying knowledge. Also, several strategies may have been used that compound each other and make the effects difficult to separate, or respondents may have been using strategies that were not communicated to the interviewer.

Indeed, the low Spearman's rank correlations for Group 1 between the cloze procedure completion scores and the number of places located on Sketch Map $2(r=-0.005)$ and for Group 2 between the projective convergence and orientation 
TABLE 6

Investigating the Role of Strategy and Metacognition on the Cloze Procedure Test

\begin{tabular}{|c|c|c|c|c|c|c|c|c|c|c|c|}
\hline Respondents & $\begin{array}{c}\text { N-Degree } \\
\text { Places } \\
\text { Located }\end{array}$ & $\begin{array}{c}\text { N-Degree } \\
\text { of Common } \\
\text { Strategies } \\
\text { (+ Other) }\end{array}$ & C1 & $C 2$ & C3 & $C 4$ & C5 & C6 & $C 7$ & $C 8$ & $\begin{array}{c}\text { Meta- } \\
\text { cognition }\end{array}$ \\
\hline M2 & 100 & 1 & & & $x$ & & & & & & + \\
\hline M1 & 86.67 & 1 & & & & & & & & $x$ & $=$ \\
\hline F1 & 80 & $3(1)$ & $x$ & $x$ & $x$ & & & & & & $=$ \\
\hline M10 & 73.33 & 1 & $x$ & & & & & & & & $=$ \\
\hline F3 & 66.67 & $1(2)$ & & & & & & & & $x$ & - \\
\hline F10 & 66.67 & 4 & $x$ & & $x$ & & $x$ & & & $x$ & - \\
\hline F5 & 60 & 2 & & & & & $x$ & & & $x$ & $=$ \\
\hline $\mathrm{F7}$ & 60 & 1 & & & & & & & & $x$ & $=$ \\
\hline F9 & 60 & 3 & $x$ & & $x$ & & $x$ & & & & + \\
\hline M6 & 60 & 2 & & & $x$ & & $x$ & & & & - \\
\hline M7 & 60 & $1(1)$ & & & $x$ & & & & & & $=$ \\
\hline F4 & 53.33 & $1(1)$ & & & & & & & & $x$ & + \\
\hline F8 & 53.33 & $3(1)$ & & & $x$ & & $x$ & & & $x$ & - \\
\hline M3 & 53.33 & $1(1)$ & & & & & & & & $x$ & $=$ \\
\hline M5 & 53.33 & $2(1)$ & $x$ & & & & $x$ & & & & - \\
\hline M8 & 53.33 & 1 & $x$ & & & & & & & & + \\
\hline M9 & 53.33 & 2 & $x$ & & $x$ & & & & & & $=$ \\
\hline F2 & 40 & 2 & $x$ & & $x$ & & & & & & $=$ \\
\hline M4 & 40 & $4(1)$ & $x$ & & $x$ & $x$ & & & & $x$ & $=$ \\
\hline F6 & 20 & 1 (1) & & & $x$ & & & & & & - \\
\hline
\end{tabular}

NOTE: + Respondent thought he/she had a good knowledge of Swansea; = respondent thought he/she had a reasonable knowledge of Swansea; - respondent thought he/she had a poor knowledge of Swansea.

specification results $(r=0.317)$ suggest that either the tasks are measuring different knowledge bases, or the tests are introducing bias effects into the results through the tasks set. The interviews tended to indicate that the same knowledge base is being interrogated; so bias effects are the most likely explanation, with these most probably introduced because of incomplete knowledge instituting large residuals. For example, on the projective convergence test if the location of one place is not known, say Hendrefoilan, then it will be very difficult for respondents to estimate the distance and direction to and from Hendrefoilan. In fact, the respondents will be introducing large 
TABLE 7

Investigating the Role of Strategy and Metacognition on the Projective Convergence Test

\begin{tabular}{|c|c|c|c|c|c|c|c|c|c|c|c|}
\hline Respondents & $\begin{array}{l}\text { N-Degree } \\
\text { Places } \\
\text { Located }\end{array}$ & $\begin{array}{l}\text { N-Degree } \\
\text { of Common } \\
\text { Strategies } \\
\text { (+ Other) }\end{array}$ & $C 1$ & $C 2$ & $C 3$ & $C 4$ & C5 & C6 & $C 7$ & $C 8$ & $\begin{array}{c}\text { Meta- } \\
\text { cognition }\end{array}$ \\
\hline F18 & 88.21 & 4 & $x$ & & $x$ & & $x$ & & & $x$ & $=$ \\
\hline $\mathrm{F} 17$ & 86.37 & $4(1)$ & $x$ & & $x$ & & $x$ & $x$ & & & + \\
\hline $\mathrm{F} 12$ & 78.90 & $5(1)$ & $x$ & $x$ & $x$ & & & $x$ & $x$ & & + \\
\hline F15 & 78.68 & 2 & $x$ & & & & & $x$ & & & - \\
\hline M13 & 76.79 & $2(1)$ & $x$ & & $x$ & & & & & & $=$ \\
\hline F13 & 70.17 & $2(1)$ & $x$ & & $x$ & & & & & & $=$ \\
\hline F16 & 68.83 & 1 & $x$ & & & & & & & & $=$ \\
\hline M20 & 66.30 & $5(2)$ & $x$ & $x$ & $x$ & $x$ & $x$ & & & & $=$ \\
\hline $\mathrm{F} 20$ & 63.66 & $3(1)$ & $x$ & & $x$ & $x$ & & & & & $=$ \\
\hline M18 & 63.62 & 3 & $x$ & $x$ & $x$ & & & & & & $=$ \\
\hline F14 & 62.99 & 1 & $x$ & & & & & & & & $=$ \\
\hline M16 & 62.20 & $4(1)$ & $x$ & $x$ & & $x$ & & & & $x$ & $=$ \\
\hline F11 & 52.09 & $3(1)$ & $x$ & $x$ & $x$ & & & & & & $=$ \\
\hline M12 & 45.36 & $3(1)$ & $x$ & & $x$ & & $x$ & & & & + \\
\hline M19 & 35.13 & 1 & $x$ & & & & & & & & - \\
\hline F19 & 33.88 & $1(1)$ & & & $x$ & & & & & & $=$ \\
\hline M17 & 33.67 & 1 & & & & & & & $x$ & & - \\
\hline M14 & 21.24 & $2(2)$ & $x$ & & $x$ & & & & & & $=$ \\
\hline M11 & 18.13 & 1 & $x$ & & & & & & & & $=$ \\
\hline M15 & 17.13 & $2(1)$ & & & $x$ & & $x$ & & & & - \\
\hline
\end{tabular}

NOTE: + Respondent thought he/she had a good knowledge of Swansea; = respondent thought he/she had a reasonable knowledge of Swansea; - respondent thought he/she had a poor knowledge of Swansea.

residuals through guessing, essentially entering random data into the analysis. These residuals might not be entered into the orientation specification test because the respondent can still work out the answer in relation to the two places he or she does know; enough information is supplied for them to be able to negotiate the problem of incomplete knowledge.

Table 5, Table 6, Table 7, and Table 8 also show the metacognition of the respondents, and represents how well they thought they knew the Swansea area. The plus $(+)$ symbol is used to represent respondents who thought they had a good knowledge of Swansea; the equals $(=)$ symbol is used to 
TABLE 8

Investigating the Role of Strategy and Metacognition on the Orientation Specification Test

\begin{tabular}{|c|c|c|c|c|c|c|c|c|c|c|c|}
\hline Respondents & $\begin{array}{c}\text { N-Degree } \\
\text { Places } \\
\text { Located }\end{array}$ & $\begin{array}{c}\text { N-Degree } \\
\text { of Common } \\
\text { Strategies } \\
\text { (+ Other) }\end{array}$ & C1 & C2 & C3 & C4 & C5 & C6 & $C 7$ & $C 8$ & $\begin{array}{c}\text { Meta- } \\
\text { cognition }\end{array}$ \\
\hline$F 11$ & 95 & $2(2)$ & $x$ & & & & & & & $x$ & $=$ \\
\hline F14 & 95 & $2(1)$ & $x$ & $x$ & & & & & & & $=$ \\
\hline F18 & 95 & $2(3)$ & $x$ & $x$ & & & & & & & $=$ \\
\hline M20 & 95 & $2(3)$ & $x$ & $x$ & & & & & & & $=$ \\
\hline M16 & 90 & $2(1)$ & $x$ & & & & & & & $x$ & $=$ \\
\hline $\mathrm{F} 12$ & 85 & 2 (1) & $x$ & & & & & $x$ & & & + \\
\hline F17 & 85 & $3(1)$ & $x$ & $x$ & & & & $x$ & & & + \\
\hline F20 & 85 & $2(2)$ & $x$ & $x$ & & & & & & & $=$ \\
\hline M11 & 80 & $3(1)$ & $x$ & $x$ & & & & $x$ & & & $=$ \\
\hline M12 & 80 & $1(1)$ & & $x$ & & & & & & & + \\
\hline$F 19$ & 65 & $2(2)$ & & & $x$ & & $x$ & & & & $=$ \\
\hline M13 & 65 & 1 & & $x$ & & & & & & & $=$ \\
\hline F15 & 60 & $2(2)$ & $x$ & & & & & & & $x$ & - \\
\hline F16 & 55 & $2(2)$ & $x$ & & & & & & & & $=$ \\
\hline M19 & 45 & $3(1)$ & & $x$ & & & $x$ & $x$ & & & - \\
\hline $\mathrm{F} 13$ & 40 & $1(1)$ & & $x$ & & & & & & & - \\
\hline M15 & 40 & 1 & $x$ & & & & & & & & - \\
\hline M18 & 40 & $1(3)$ & $x$ & & & & & & & & $=$ \\
\hline M14 & 20 & $2(2)$ & $x$ & & & & & & & $x$ & $=$ \\
\hline
\end{tabular}

NOTE: + Respondent thought he/she had a good knowledge of Swansea; = respondent thought he/she had a reasonable knowledge of Swansea; - respondent thought he/she had a poor knowledge of Swansea.

represent a reasonable or okay knowledge; and the minus (-) symbol is used to represent poor knowledge. ANOVA tests indicated that the respondents' metacognition did not lead to more accurate results on any of the tests (sketch map test, $f=$ $3.00, p=.077$; cloze procedure test, $f=0.73, p=.496$; projective convergence test, $f=1.56, p=.240$; orientation specification test, $f=3.30, p=.063$ ). It seems that the respondents were inaccurate in judging the quality of their knowledge and suggests that confidence in ability has little relation to actual ability. The reason for the lack of correspondence between metacognition and ability is unknown and may be reliant on personality factors. 


\section{CONCLUSION}

The purpose of the research reported in this article was to conduct an exploratory, phenomenological examination of how individuals think about the tasks set and arrive at solutions when completing cognitive mapping exercises. The aims were to discover the strategies adopted and how they differed, and to explore whether the adoption of certain strategies led to better (more accurate) results. It was noted that the results from this study are tenuous and they cannot easily be validated because there are alternative possibilities for outcomes. For example, the relationship between the amount of knowledge and the strategies of thought is not clear. It may be the case that using different strategies of thought on the same knowledge base will not cause any differences, but that different strategies are associated with different amounts or form of knowledge, so that differences occur because of knowledge not strategy. Also, respondents could have been using strategies that they did not report or could not fully articulate. The introspective methodology is reliant on the assumption that the strategies of spatial thought are available for conscious inspection. In addition, it may well be the case that respondents used multiple strategies to complete each task and the one articulated by the respondent may or may not have been critical in generating the answer that was given. It is also possible that the interviewing altered strategies of thought or implemented the uptake of strategies not commonly used.

The results do allow, however, an introspective insight into the way in which we think about geographically based tasks. It is clear that there is a range of common and task-specific strategies that can be used to process cognitive map knowledge while performing geographic or spatial tasks, and that cognitive map knowledge is held in a variety of mediums. This knowledge may be manipulated, transposed, and constructed to aid the process of spatial thought. There is clearly room for more research concerning the form and structure of cognitive map knowledge and its relationship to the task demands of spatial and geographic exercises. At present, data collection 
methods suffer from a number of construct (is the test measuring what it is supposed to without introducing bias?) and convergent validity (do different tests designed to measure the same phenomena produce similar results?) problems (see Montello, 1991). Unless these validities are strengthened, few definitive conclusions can be drawn from cognitive mapping research, leading to little utility in wayfinding, planning, education, and map design. From such qualitative introspection, it is hoped that more rigorous quantitative exercises can be designed to explore the mental processes involved in spatial thought, the relationships between strategy, knowledge, and task, and the effectiveness of the tests for predicting spatial behavior.

\section{REFERENCES}

Beck, R. J., \& Wood, D. (1976). Cognitive transformations from urban geographic fields to mental maps. Environment and Behavior, 8, 199-238.

Blades, M. (1990). The reliability of data collected from sketch maps. Joumal of Environmental Psychology, 10, 327-339.

Boyle, M. J., \& Robinson, M. E. (1979). Cognitive mapping and understanding. In D. T. Herbert \& R. J. Johnston (Eds.), Geography and the urban environment: progress in research and applications (Vol. 2, pp. 59-82). London: Wiley.

Bryant, K. J. (1984). Methodological convergence as an issue within environmental cognition research. Joumal of Environmental Psychology, 4, 43-60.

Buttenfield, B. P. (1986). Comparing distortion on sketch maps and MDS configurations. Professional Geographer, 38, 238-246.

Cadwallader, M. T. (1979). Problems in cognitive distance and their implications to cognitive mapping. Environment and Behavior, 11, 559-576.

Catling, S. (1978). Cognitive mapping exercises as a primary geographical experiences. Teaching Geography, 3, 120-123.

Coolican, H. (1990). Research methods and statistics in psychology. London: Hodder \& Stoughton.

Day, R. A. (1976). Urban distance cognition: review and contribution. Australian Geographer, 13, 193-200.

Downs, R. M., \& Stea, D. (Eds.). (1973a). Image and environment. Chicago: Aldine.

Downs, R. M., \& Stea, D. (1973b). Theory. In R. M. Downs \& D. Stea (Eds.), Image and environment (pp. 1-8). Chicago: Aldine.

Edwards, G. (1991). Spatial knowledge for image understanding. In D. M. Mark \& A. U. Frank (Eds.), Cognitive and linguistic aspects of geographic space (pp. 35-44). Norwell, MA: Kluwer Academic Publishers. 
Ericsson, K. A., \& Simon, H. A. (1980). Verbal reports as data. Psychological Review, $87,215-251$.

Evans, G. W., \& Pezdek, K. (1980). Cognitive mapping: knowledge of real-world distance and location information. Joumal of Experimental Psychology: Human Leaming and Memory, 6, 13-24.

Evans, G. W., Fellows, J., Zorn, M., \& Doty, K. (1980). Cognitive mapping and architecture. Joumal of Applied Psychology, 65, 474-478.

Foley, J. E., \& Cohen, A. J. (1984). Working mental representations of the environment. Environment and Behavior, 16, 713-729.

Foos, F. W. (1980). Constructing maps from sentences. Joumal of Experimental Psychology: Human Leaming and Memory, 6, 25-38.

Gärling, T., \& Evans, G. W. (Eds.). (1991). Environment, cognition and action-an integrated approach. New York: Oxford University Press.

Gärling, T., \& Golledge, R. G. (Eds.). (1993). Behaviour and environment: Psychological and geographical approaches. London: North Holland.

Gerber, R., \& Kwan, T. (1994). A phenomenological approach to the study of preadolescents' use of maps in a wayfinding exercise in a suburban environment. Joumal of Environmental Psychology, 14, 265-280.

Golledge, R. G. (1992). Place recognition and wayfinding: Making sense of space. Geoforum, 23, 199-214.

Golledge, R. G. (1993). Geographical perspectives on spatial cognition. In T. Gärling \& R. G. Golledge (Eds.), Behaviour and environment: Psychological and geographical approaches (pp. 16-46). North Holland: Elsevier.

Golledge, R. G., Gale, N., \& Richardson, G. D. (1987). Cognitive maps of cities II: Studies of selected populations. National Geographical Joumal of India, 33, 1-16.

Hardwick, D. A., McIntyre, C. W., \& Pick, H. L. (1976). The content and manipulation of cognitive maps in children and adults. Monographs of the Society for research in Child Development, 41, 1-55.

Hart, R. (1979). Children's experience of place. New York, Irvington.

Howard, R. B., Chase, S. D., \& Rothman, M. (1973). An analysis of four measures of cognitive maps. In W.F.E. Preisner (Ed.), Environmental design research 1 (pp. 254264). Stroudsberg, PA: Dowden, Hutchinson, \& Ross.

Humphreys, J. S. (1990). Place learning and spatial cognition: A longitudinal study of urban newcomers. Tijdschrift Voor Econmicshe en Sociale Geografie, 81, 364-380.

Jackson, P., \& Smith, S. (1984). Exploring social geography. London: Allen \& Unwin.

Jacobson, R. D. (1992). Spatial cognition through tactile mapping. Swansea Geographer, 29, 79-88.

Kirasic, K. C., Allen, G., \& Siegel, A. W. (1984). Expression of configurational knowledge of large scale environments: Students' performance of cognitive tasks. Environment and Behavior, 16, 687-712.

Kirasic, K. C. Siegel, A. W., \& Allen, G. (1981). The development of basic processes in cognitive mapping: Recognition in context memory. Child Development, 51, $302-$ 305.

Kitchin, R. M. (1994a). Cognitive maps: What are they and why study them? Joumal of Environmental Psychology, 14, 1-19.

Kitchin, R. M. (1994b). CMAP: Cognitive Mapping Analysis Package. Swansea: Department of Geography, University of Wales-Swansea. 
Kitchin, R. M. (1995). Issues of validity and integrity in cognitive mapping research: Investigating configurational knowledge. Unpublished doctoral dissertation, University of Wales-Swansea.

Kitchin, R. M. (in press). Increasing the integrity of cognitive mapping research: Appraising conceptual schemata of environment-behavior interaction. Progress in Human Geography.

Landau, B., \& Jackendoff, R. (1993). "What" and "where" in spatial language and spatial cognition. Behavioral and Brain Sciences, 16, 217-265.

Liben, L. (1981). Spatial representation and behavior: multiple perspectives. In L. S. Liben, A. Patterson, \& N. Newcombe (Eds.), Spatial representation and behavior across the life span. New York: Academic Press.

Lloyd, R. (1989). Cognitive maps: encoding and decoding information. Annals of the Association of American Geographers, 79, 101-124.

Lynch, K. (1960). The image of the city. Cambridge, MA: MIT Press.

Lynch, K. (1976). Preface. In G. T. Moore \& R. G. Golledge (Eds.), Environmental knowing (pp. v-viii). Stroudsberg, PA: Dowden, Hutchinson, \& Ross.

Magana, J. R., Evans, G. W., \& Romney, A. K. (1981). Scaling techniques in the analysis of environmental cognition data. Professional Geographer, 33, 294-301.

Mark, D. M., \& Frank, A. U. (Eds.), (1991). Cognitive and linguistic aspects of geographic space. Norwell, MA: Kluwer Academic Publishers.

Matthews, M. H. (1984). Cognitive maps: a comparison of graphic and iconic techniques. Area, 16, 33-40.

Miller, A. (1985). Cognitive styles and environmental problem solving. Intemational Joumal of Environmental Studies, A26, 21-31.

Montello, D. R. (1991). The measurement of cognitive distance: methods and construct validity. Joumal of Environmental Psychology, 11, 101-122.

Moore, G. T., \& Golledge, R. G. (Eds.). (1976). Environmental knowing. Stroudsberg, PA: Dowden, Hutchinson, \& Ross.

Nesbitt, R. E., \& Wilson, T. D. (1977). Telling more than we can know: Verbal reports on mental processes. Psychological Review, 84, 231-259.

Newell, A., \& Simon, H. A. (1972). Human problem solving. Englewood Cliffs, NJ: Prentice Hall.

Passini, R., \& Proulx, G. (1988). Wayfinding without vision: an experiment with congenitally blind people. Environment and Behavior, 20, 227-252.

Pearce, P. L. (1981). Astudy of travelers perceptions of a section of countryside. Journal of Environmental Psychology, 1, 141-155.

Portugali, J. (Ed.). (in press). The construction of cognitive maps.

Robinson, M. E. (1974). Cloze procedure and spatial comprehension test. Area, 9, 137142.

Saarinen, T. F. (1973). Student views of the world. In R. M. Downs \& D. Stea (Eds.), Image and environment (pp. 148-161). Chicago: Aldine.

Saarinen, T. F., MacCabe, C. L. \& Morehouse, B. (1988). Sketch maps of the world as surrogates for world geographic knowledge (Discussion Paper 83-3). Tucson: University of Arizona, Dept. of Geography and Regional Development.

Spencer, C., \& Blades, M. (1993). Children's understanding of places: The world at hand. Geography, 78, 367-373.

Spencer, C. Blades, M., \& Morley, K. (1989). The child in the physical environment. Chichester, UK: Wiley. 
Taylor, H. A., \& Tversky, B. (1992). Description and depictions of environments. Memory and Cognition, 20, 483-496.

Thorndyke, P. W. (1983). Spatial cognition and reasoning. In J. Harvey (Ed.), Cognition, social behavior and the environment (pp. 137-149). Hillsdale, NJ: Lawrence Erlbaum.

Thorndyke, P. W., \& Hayes-Roth, B. (1982). Differences in spatial knowledge acquired from maps and navigation. Cognitive Psychology, 14, 560-589.

Tobler, W. R. (1965). Computation of correspondence of geographic patterns. Papers and Proceedings of the Regional Science Association, 15, 131-139.

Tobler, W. R. (1976). The geometry of mental maps. In R. G. Golledge \& G. Rushton (Eds.), Spatial choice and spatial behavior (pp. 69-82) Columbus: Ohio State University Press.

Tuan, Y-F. (1975). Images and mental maps. Annals of the Association of American Geographers, 65, 205-213.

Vanetti, E. J., \& Allen, G. L. (1988). Communicating environmental knowledge-The impact of verbal and spatial abilities on the production and comprehension of route directions. Environment and Behavior, 26, 667-682.

Wood, D., \& Beck, R. (1976). Talking with Environmental A: An experimental mapping language. In G. T. Moore \& R. G. Golledge (Eds.), Environmental knowing (pp. 351361). Stroudsberg, PA: Dowden, Hutchinson, \& Ross. 